\title{
Cost-Utility of Rotator Cuff Repair Surgery by Open and Arthroscopic Techniques: Study Protocol for a Randomized Clinical Trial
}

RAFAEL PIERAMI ( $\square$ rafael_pierami@hotmail.com )

Hospital Israelita Albert Einstein https://orcid.org/0000-0002-1745-4362

\section{Eliane Antonioli}

Hospital Israelita Albert Einstein

Isadora Oliveira

Hospital Israelita Albert Einstein

Isabela Castro

Hospital Israelita Albert Einstein

Felipe Manente

Faculdade de Medicina Albert Einstein

Paula Fairbanks

Faculdade de Medicina Albert Einstein

\section{Eduardo Carrera}

Hospital Israelita Albert Einstein

\section{Bruno Matsumura}

Hospital Israelita Albert Einstein

Mario Lenza

Hospital Israelita Albert Einstein

\section{Study protocol}

Keywords: rotator cuff, surgery, arthroscopy, open repair, cost-effectiveness, QALY

Posted Date: July 29th, 2020

DOI: https://doi.org/10.21203/rs.3.rs-19377/v1

License: (c) (i) This work is licensed under a Creative Commons Attribution 4.0 International License.

Read Full License 


\section{Abstract}

Background: Shoulder pain is one of the most common musculoskeletal conditions in orthopedic practice. Rotator cuff injuries account for up to $70 \%$ of pain in the shoulder girdle. However, there is still no consensus on the best surgical treatment of patients with degenerative rotator cuff injuries, considering cost-effectiveness and cost-utility analysis between the open and arthroscopic methods of rotator cuff repair. The objective of this trial is to compare the efficacy, cost-effectiveness and cost-utility of open and arthroscopic procedure for rotator cuff repair.

Methods: The trial is a two-group, parallel design, randomized controlled study. A total of 100 patients with symptomatic rotator cuff lesion will be allocated to repair surgery and randomized by either open or arthroscopic technique in a 1:1 ratio, considering smoking (yes or no), lesion size (less than $3 \mathrm{~cm}$ or more than $3 \mathrm{~cm}$ ) and diabetes (present or absent) as stratification factors. All patients will be included in the same rehabilitation program after the intervention. The primary outcome measure will be the ConstantMurley score at 48 weeks post-surgery. Secondary outcomes include cost-effectiveness, cost-utility, pain, complications and clinical analysis, using the EuroQol 5-D3L, the simple shoulder test (SST), Visual Analogue Pain Scale (VAS), integrity of the repair evaluated through magnetic resonance imaging, complications and failures of the proposed methods. Theses outcomes will also be measured continuously for 48 weeks after the intervention. For the cost-effectiveness analyses, we will use the VAS and the Constant-Murley Score as measures of effectiveness; for the cost-utility analyses, we will use the EuroQol- 5D-3L as a measure of utility in terms of incremental cost per quality-adjusted life-years (QALY).

Discussion: There are two options of the rotator cuff repair surgery, open and arthroscopic and, both procedure present good clinical results. However, considering that the arthroscopic surgery present supposedly higher costs, is necessary to understand if these costs present a best cost-effectiveness and cost-utility for the patient.

Trials registration: ClinicalTrials.gov register: NCT04146987. Registered October 31, 2019

\section{Introduction}

\section{4a. Background and Rationale}

Musculoskeletal injuries are a major cost to the healthcare system. In 2004, 30\% of the North American population had some kind of musculoskeletal disorder that required medical treatment; between 2002 and 2004, the estimated cost of treating these changes was $\$ 510$ billion. Shoulder diseases represent the third most common cause of these changes, behind only spinal and knee disorders [1], [2].

An evaluation of the primary health care system in Cambridge, United Kingdom, showed that the average frequency of shoulder pain was 9.5 per 1,000 individuals [3]. Of these, $86 \%$ had rotator cuff tendinopathy. North American data estimate that approximately 4.5 million patients annually seek medical attention due to shoulder pain; of these, two million have some symptoms related to the rotator cuff. About 
250,000 rotator cuff repair surgeries are performed annually in the United States of America (US), and with the continued increase in life expectancy and aging, there is a tendency to increase this number [1], [2].

The rotator cuff is a group of four muscles and their tendons that act to stabilize the shoulder and allow for its extensive range of motion. Four muscles and their attached tendons make up the rotator cuff: the subscapularis, supraspinatus, infraspinatus, and teres minor. The long portion of the biceps tendon also contributes to cuff function, which is to stabilize the humeral head in the glenoid cavity, preventing superior migration of the humeral head [4].

The possible injuries range from tendon degeneration (tendinosis/tendinopathy), through partial tear (articular, interstitial or bursal), to complete tear. Diagnosis is made by associating history and physical examination along with imaging methods, and magnetic resonance imaging (MRI) is considered the method of choice [5]-[13].

Currently, the indication for surgical treatment is based on the persistence of symptoms and/or the degree of muscle weakness and/or size of the tear, after a time of conservative treatment. In general, when opting for surgery, imaging can assist in the planning of surgical treatment, since it allows measuring the extent of the tear (partial or total) and discriminating which tendons are involved (supraspinatus, infraspinatus, etc.).

Treatment of rotator cuff tear depends on the type of injury, the patient's functional capacity, age, and the presence of symptoms. In general, tendon degeneration and partial tears are treated non-surgically, with physiotherapy, injections and analgesic medications. Complete and incomplete tears that did not respond well to conservative treatment, however, should be treated surgically [8], [14]-[16].

Among the surgical options, the open method is still considered the gold standard, with good or excellent results in over $90 \%$ of cases [17]-[19]. Due to arthroscopy and the evolution of arthroscopic instruments and implants in the last two decades, the arthroscopic repair technique has gained space and is widely used in our country. Some studies [17]-[20] did not show superiority of one technique over another in terms of clinical outcomes. On the other hand, since the cost of arthroscopic surgery is supposedly higher, due to the required equipment, it is important to establish which option has the best cost-utility ratio. Other published studies suggested that the open method is superior than the arthroscopic method in relation to cost-utility [21]-[23].

\section{4b. Objectives}

Despite the high incidence of rotator cuff injury, there is no consensus about the best method of repair, neither which method has the cost-effectiveness and cost-utility ratio. Therefore, the present study aims to compare the open and arthroscopic methods for rotator cuff repair and determine which presents the best cost-effectiveness ratio.

\section{Trial Design}


The trial will be a prospective randomized controlled clinical trial.

\section{Methods}

This randomized controlled trial will follow the Consolidated Standards of Reporting Trials (CONSORT) Statement [24]; it will be performed at Hospital Alvorada Moema (Shoulder and Elbow Surgery Center of Excellence), São Paulo, Brazil, the cost analysis will be performed by Hospital Israelita Albert Einstein team, São Paulo, Brazil. The study has been approved by the local Research Ethics Committee (CAAE 19182619.3.1001.0071). The project is registered in the ClinicalTrials.gov database (NCT04146987 https://clinicaltrials.gov/ct2/show/NCT04146987?term=NCT04146987\&draw=2\&rank=1).

\section{6a. Sample size}

The sample size estimate was obtained to detect differences between the open and arthroscopic repair groups in relation to the primary outcome of the study, Constant-Murley Score (CM) instrument after the intervention. Kukkone's et al. 2013 study [25] estimated the clinically important minimal difference in CM score in 10.4 points in patients with rotator cuff rupture after 3 months of surgical treatment by the arthroscopic method. The estimated sample size of 45 patients per group, total of 90 patients, would reach $90 \%$ power to detect a 10.4 difference between the groups in the $\mathrm{CM}$ instrument post-operative score with a standard deviation of up to 15 points with a significance level of $5 \%$ using a t-Student test. Predicting a loss of around $10 \%$ at 12 months of follow-up we aim to recruit 50 patients per group (PASS software [26]).

\section{6b. Inclusion criteria}

All patients eighteen years of age or older, presenting with complete rotator cuff injury or a high-grade partial rotator cuff injury, symptomatic, where conservative therapy failed (maintenance of pain and disability after conservative treatment), or the patient could not support the non-surgical treatment. All patients ought not to have any medical contraindications for surgery, have a good understanding of the Portuguese language, agree to participate in the study and sign the Informed Consent Form.

\section{6c. Exclusion criteria}

Patients with previous shoulder surgery, previous fractures in the affected shoulder, those with passive range of motion limitation (joint stiffness with an elevation of 90 degrees or less), radiographic signs of glenohumeral osteoarthritis or neurologic injury will be excluded. Patients will also be excluded if they do not wish to participate or are unable to understand or sign the informed consent form (due to conditions such as cognitive impairment, or mental illness) or if there are any conditions that contraindicate any of the surgical methods.

\section{7c. Randomization and allocation}


After eligibility assessment, all patients will be informed about the nature and purpose of the study and will only be included after signing the informed consent form, that will be obtained by the surgeon that evaluated the patient and indicated the surgery.. Patients will be consecutively allocated to one of two proposed treatment methods: open rotator cuff repair or arthroscopic rotator cuff repair (FIGURE 1). The software $\mathrm{R}$ was used to generate a randomization list, considering 100 patients to be included in the study and the same probability of allocation for both methods of surgery (open and arthroscopic repair). The variables will be: smoking (yes or no), the size of the lesion ( $\leq 3 \mathrm{~cm}$ or $>3 \mathrm{~cm}$ ) and diabetes (present or absent). Randomization will be performed by the REDCap platform (Research Electronic Data Capture - Vanderbilt University)[27][28] after the patient is anesthetized and prepared for the surgery. A person not associated with the study will open the software and acquire one of the two techniques possible and tell the surgeon who will perform the surgery.

\section{7d. Blinding}

Due to the type of interventions, neither participants nor treatment providers can be blinded to treatment allocation. The outcome assessment of the primary and secondary outcomes (Constant-Murley; EuroQol; VAS and SST), patient-reported outcomes, will not be blind. One of the authors (RP) will assess all other clinical outcomes. All primary and secondary outcomes will be assessed at baseline, 6, 24 and 48 weeks, except for the VAS which will also be assessed at hospital discharge, 1, 2 and 4 weeks. The statisticians conducting the analyses will be blinded to the treatment status until the analyses are completed.

\section{Intervention methods}

Five surgeons with at least four years of surgical technique experience will participate in this study (EFC, MTCA, RP, BAM, VR). Also, the residents of shoulder and elbow surgery, as well as the residents of Orthopedics and Traumatology from Hospital Alvorada Moema and residents in shoulder and elbow surgery at Albert Einstein Hospital may participate in surgeries.

Open surgery: patients will be positioned in a beach chair position with the affected limb pending off the table, allowing manipulation and full range of motion range. After asepsis, antisepsis, and placement of sterile surgical fields, an anterolateral incision will be made in the shoulder in question; the deltoid muscle belly will be gently divided along its fibers until exposure of the subdeltoid / subacromial bursa, which will be partially excised for exposure of the subacromial space and rotator cuff tendons. After mobilization and release of the ruptured tendons and debridement of the rotator cuff footprint, the tendon repair to the bone will be performed using $5.5 \mathrm{~mm}$ metal anchors, according to the preference and technique chosen by the surgeon. In all cases, the release of the coracoacromial ligament and acromioplasty will be performed.

Arthroscopic Technique: the patients will be positioned in lateral decubitus position, with the limb to be operated attached to a skin traction device, which through a traction post and 7 kilograms $(\mathrm{kg})$, will maintain the shoulder in the following position: abduction of 30 to 60 degrees and flexion of 20 to 30 degrees. After asepsis, antisepsis, and placement of impermeable sterile surgical fields, a posterolateral 
incision will be made in the shoulder for optic introduction, with a $50 \mathrm{mmHg}$ pressure pump and a 0.90 flow, and inspection of the glenohumeral joint. If necessary, an anterior accessory portal will be performed for intra articular instrumentation. After joint inspection, the optic will be introduced into the subacromial space with detachment of the subacromial and subdeltoid bursa with the trocar. After visualizing the lesion, an accessory lateral portal will be performed. With the use of shaver blades, partial bursectomy will be performed and any adherence to the tendon stumps will be released, as well as debridement of the rotator cuff footprint. The tendon will then be reinserted to the bone using metallic $5.5 \mathrm{~mm}$ anchors, according to the preference of each surgeon. The technique used, as well as the suture configuration and type of knot used, will be defined by the surgeon, according to his preference. After tendon repair, the coracoacromial ligament will be released, as well as acromioplasty.

\section{Postoperative rehabilitation}

All patients will undergo the same postoperative rehabilitation protocol: use of Velpeau sling for 6 weeks; pendulum exercises from the second week; active movement and recovery of the range of motion from the sixth week and strengthening from the twelfth week.

The patients will be oriented to perform home exercises and, as well, to be assisted by a physiotherapist twice a week from the sixth week of surgery and on. It is expected at the end of treatment the need of about thirty sessions of physical therapy.

\section{Outcomes assessment}

Study data will be collected and managed using REDCap (Research Electronic Data Capture) hosted at Hospital Israelita Albert Einstein [27][28]. REDCap is a secure, web-based software platform designed to support data capture for research studies, providing: 1 ) an intuitive interface for validated data capture; 2 ) audit trails for tracking data manipulation and export procedures; 3 ) automated export procedures for seamless data downloads to common statistical packages; and 4) procedures for data integration and interoperability with external sources.

All study participants will be evaluated preoperatively, at the hospital discharge and 1, 2, 6, 24 and 48 weeks after the intervention. The Constant-Murley score, Visual Analogue Scale, EuroQol-5D-3L and the Simple Shoulder Test validated to the Portuguese language questionnaires will be filled out by the patient and assessed by evaluators to the assigned intervention.

To prevent loss of follow-up all the patients will be monitored by REDCap software and alerts will be sent to each patient near time points defined by the investigators. One week before every medical consultation and at the twelfth week, regarding the rehabilitation process. If the patient fails to fill any questionnaire or does not attend the medical consultations, he will be contacted by phone and e-mail.

\section{0a. Primary outcome}


The Brazilian Portuguese Version of the Constant-Murley Score (CM) [29] will be measured preoperatively at 6, 24 and 48 weeks after the intervention. Research assistants (not blinded to the study aim) will ask the patients to fill in the validated CM form for the Portuguese language and measure the range of motion with a goniometer. The CM scale covers different domains of shoulder function (pain, activities of daily living, range of motion and power), punctuating each of them; it ranges from 0 to 100 , with higher scores indicating better function.

\section{0b. Secondary outcomes}

EuroQol-5D-3L (European Quality of Life), a generic score developed to describe health-related quality of life [24] will also be assessed preoperatively, at 6, 24 and 48 weeks postoperatively. This score includes five health domains: mobility, self-care, usual activities, pain/discomfort, and anxiety/depression; each domain has 3 levels: no problem; some problems and extreme problems. In addition, the EuroQol-5D-3L has a visual analog scale where the participant assigns a value between zero and one hundred to his or her own health condition [30]. At the end of its application, EuroQol-5D-3L will provide a unique numerical value that can be used for longitudinal comparison between different time periods.

Clinical outcomes will also be assessed by the Simple Shoulder Test (SST), validated for Portuguese [31], preoperatively and at 6, 24 and 48 weeks after the procedure. SST is a simple, quick and widely used questionnaire for shoulder function measurement; it consists of 12 dichotomous questions answered by the patient himself. Each positive answer (yes) is given a score; at the end of the questionnaire the percentage of positive answers (score) is made, and the higher the percentage, the better the shoulder function. Other outcomes measured will be VAS (visual analogue pain scale) at hospital discharge, 1, 2, 6, 24 and 48 weeks after the intervention. This scale allows pain intensity to be measured with maximum interobserver reproducibility; it consists of a $10 \mathrm{~cm}$ straight line with the ends determining the limits of pain sensation (no pain; worst pain ever experienced); the distance between zero (no pain) and the patient's demarcation defines the intensity of pain. Complications and failures of the proposed methods will also be assessed.

Failures will be characterized as the need for additional surgical procedures and/or change of the initially proposed procedure. Patients who, for any reason, demonstrate treatment failure or require additional interventions will be followed up and their results included in the group in which they were initially randomized, according to the intention to treat principle.

After the 48th week, all patients will be submitted to Magnetic Resonance Imaging (MRI) of the operated shoulder to evaluate the integrity and healing of the repair performed.

\section{0c. Cost-effectiveness}

Cost-effectiveness and cost-utility analyses will be assessed by the estimate of direct and indirect costs to the private healthcare system at 48 weeks. The perspective adopted in the study will be the social costs, the direct and indirect medical costs. The set timeframe will be 48 weeks and a discount rate of $5 \%$ 
will be applied. The costs included in direct medical costs will be: hospitalization, medical fees, medication; the indirect costs: costs of absenteeism from work, which will be estimated by the patientreported number of days away from work multiplied by the average wage rate of the current year. The costs will be converted from Brazilian Reais to US dollars and brought to the cost schedule of the current year, in order to avoid that the effect of inflation on the medical inputs influences the analysis. For the cost-effectiveness analyses, the VAS and the CM will be used as measures of effectiveness. For the costutility analyses, the EuroQOL-5D-3L will be used as a measure of utility. The timetable of outcomes assessment is described on Table 1.

\section{Data analysis}

The descriptive analyzes of variables will be based on the absolute frequencies and percentages for categorical variables and summary measures as means and standard deviations or medians and quartiles, as well as minimum and maximum values for numerical variables [32]. Clinical scores will be represented by individual profile graphs separately by the surgical technique group.

The groups will be compared according to the presence of categorical clinical outcomes (failures, complications and healing integrity) by Chi-square or Fisher's exact tests, depending on the distribution observed after data collection.

For inferential analysis of numerical clinical outcomes, mixed models will be used and, if the normal distribution is not adequate, generalized mixed models will be used [33]. The models will have time effects (preoperative, 6, 24 and 48 weeks after intervention), surgical technique group (open repair or arthroscopic repair) and the interaction effect between time and group. The size of the lesion (smaller than three $\mathrm{cm}$ or larger than three $\mathrm{cm}$ ) will also be included in the models as a control variable, seeking to avoid possible biases.

The analyzes will be performed with the aid of the SPSS program [34], considering a significance level of $5 \%$.

\section{Safety}

There will be no benefit to the participant, beyond what is expected for the correction of the rotator cuff injury, expecting an improvement of pain and function of the affected shoulder. The risks of the present study are those inherent in any surgical treatment and anesthetic procedure, such as surgical wound infection, scar formation, pain, shoulder range of motion, rotator cuff tear, neurovascular injury. If any complications occur, all patients will be treated by the same surgical team until the complication is healed.

Both surgical techniques have the same goal, that is, to repair the ruptured tendon to the bone. The open technique requires a larger incision, as well as greater surgical dissection and manipulation of the deltoid muscle, which may cause greater postoperative pain and weakness of this muscle, in addition to causing 
a slightly larger scar. However, it provides great visualization and manipulation and mobilization capability of the ruptured tendon, which provides a safer and tension-free repair.

The arthroscopic technique is performed with some point-shaped cuts in the shoulder, usually three or four; due to smaller incisions, it requires less muscle manipulation, which theoretically would cause less postoperative pain and less muscle weakness of the deltoid muscle, it also has minor scars. However, this technique requires more surgeon's experience and the mobilization of the ruptured tendon(s) is limited. Using a large amount of saline may cause edema in the operated shoulder, which is usually reversed after the first 12 hours of surgery.

Finally, there is a minimal risk of loss of data confidentiality, but the responsible researcher undertakes to do everything possible to maintain data confidentiality. One of the researchers will have access to all data during the entire trial period. Any adverse event will be reported to the researchers involved and communicated to the main investigator according to the Institutional Review Boards description.

\section{Discussion}

There is no consensus about the best cost-effectiveness of surgical treatment of patients with degenerative rotator cuff injuries. Several studies [21], [22], [35], [36] suggest that the open repair method is more cost-effective than the arthroscopic method, resulting in the same clinical outcome with lower cost. Adla, Deepthi N. et. al [21] in a prospective nonrandomized study, showed that both techniques lead to the same clinical outcomes. The costs of arthroscopic surgery were higher than the open surgery, mainly due to the costs of the suture anchors, which was used only in the arthroscopic group, is important to notice that in most of the open surgeries, the repair was performed through transosseous sutures. Köse, Kamil Çağri et. al [22], in a retrospective study, also demonstrated similar clinical outcomes, although the costs of arthroscopic procedure being much higher. Importantly, the open repair technique was performed using transosseous sutures and the arthroscopic method using suture anchors and also, the open repair group required longer length hospital stay. Hui, Yik Jing et. al [35] in a retrospective cohort study, described a significantly higher cost for the arthroscopic procedure, compared to the open repair, evaluating only the in-hospital costs, but with the same clinical outcomes. However, it is important to emphasize that the open repair was performed using transosseous sutures, without suture anchors and that the arthroscopic group needed a longer surgery time. Churchill, R.S. et. al [36] using the New York Ambulatory Database System, with a total of 5,224 cuff repair surgeries, of which 1,334 open repair and 3,890 arthroscopic repair, showed that the mini-open rotator cuff repair costs significantly less than the arthroscopic repair and requires significantly less surgical time. However, no clinical outcomes have been analyzed in this study, making it impossible to determine the cost-effectiveness ratio. An elegant study Carr, A.J. et. al [37] carried out as a prospective multicenter randomized clinical trial, concluded that there is no difference in the effectiveness and cost-effectiveness between the open repair surgery and arthroscopic surgery after 24 months of follow-up, even with the higher initial costs in the arthroscopy surgery. An economic evaluation of the data from this study was carried out, showing that 
the Incremental Cost Effectiveness (ICER) was uncertain and the arthroscopic repair surgery was slightly more costly and less effective than open repair surgery.

Thus, despite the high incidence of rotator cuff injury, there is insufficient evidence to determine the best method for treating these injuries. So, the present study proposes to answer the clinical question of which method, open or arthroscopic, presents the best cost-effectiveness in the surgical treatment of rotator cuff injury. Providing conclusive, good quality evidence for and contributing to the evidence base of methods used to treat rotator cuff injuries.

\section{Trial status}

Protocol Trial version: 2 Date: 03/23/2020

Recruitment Estimated Start Date: June/2020

Recruitment Estimated End Date: June/2022

Not yet recruiting.

\section{Declarations}

\subsection{Ethics Approval and Consent to Participate}

The study has been approved by the local Research Ethics Committee (CAAE 19182619.3.1001.0071). Digital, informed consent to participate will be obtained from all participants through REDCAP.

\subsection{Consent for Publication}

Not Applicable

\subsection{Availability of Data and Materials}

The datasets used and/or analysed during the current study will be available from the corresponding author on request.

\subsection{Competing interests}

The authors declare that they have no competing interests.

\subsection{Fundings}

This study is supported by Fundação de Amparo à Pesquisa do Estado de São Paulo (FAPESP 2019/02159-3).

\subsection{Author's contributions}


$\mathrm{ML}$ is the Chief Investigator; he conceived the study, led the proposal and protocol

development.

$\mathrm{RP}$ is the lead trial methodologist and helped in the study conceiving and development

EA contributed to study design and to development of the proposal.

IO contributes to study design related to QALY

IQC is responsible for cost-analysis

FM helped in the English translation and registration/publication of the trial

PF helped in the English translation and registration/publication of the trial

EFC helped in the study conceiving and development

BAM helped in the study conceiving and development

All authors read and approved the final manuscript.

\subsection{Acknowledgements}

Not applicable

\section{Dissemination policy}

All the authors are committed and agree to publish the full results of the research, despite the final results.

\section{Abbreviations}

CONSORT: Consolidated standards of reporting trials; VSA: Visual analogue scale; MRI: magnetic resonance imaging; QALY: quality-adjusted life years; CM: Constant-Murley Score; SST: Simple Shoulder Test

\section{References}

1. lii RCM, et al., "The Societal and Economic Value of Rotator Cuff Repair," pp. 1993-2000, 2013.

2. Kuye IO, Jain NB, Warner L, Herndon JH, Warner JJP. "Economic evaluations in shoulder pathologies: A systematic review of the literature," J. Shoulder Elb. Surg., 2012.

3. Ostör AJK, Richards CA, Prevost AT, Speed CA, Hazleman BL. "Diagnosis and relation to general health of shoulder disorders presenting to primary care.," Rheumatology (Oxford)., 2005.

4. Favard L, Bacle G, Berhouet J. "Rotator cuff repair.," Joint. Bone. Spine, 2007. 
5. Tempelaere C, et al. Dynamic Three-Dimensional Shoulder Mri during Active Motion for Investigation of Rotator Cuff Diseases. PLoS One. 2016;11(7):e0158563.

6. Sela Y, et al. Rotator cuff tears: correlation between geometric tear patterns on MRI and arthroscopy and pre- and postoperative clinical findings. Acta Radiol. 2015;56(2):182-9.

7. Teefey SA, Rubin DA, Middleton WD, Hildebolt CF, Leibold RA, Yamaguchi K. "Detection and Quantification of Rotator Cuff Tears: Comparison of Ultrasonographic, Magnetic Resonance Imaging, and Arthroscopic Findings in Seventy-One Consecutive Cases," J. Bone Jt. Surg. - Ser. A, 2004.

8. Jason AOG, Hsu Steven E, Lippitt B, Frederick A, Matsen III, "Rockwood and Matsen's The Shoulder, 5th Edition: The Rotator Cuff," in Rockwood and Matsen's The Shoulder, 5th Edition, 5th ed., Elsevier, 2016, pp. 651-719.

9. Yamakawa S, Hashizume H, Ichikawa N, Itadera E, Inoue H. "Comparative studies of MRI and operative findings in rotator cuff tear," Acta Med. Okayama, 2001.

10. Roy JS, et al., "Diagnostic accuracy of ultrasonography, MRI and MR arthrography in the characterisation of rotator cuff disorders: A systematic review and meta-analysis," British Journal of Sports Medicine. 2015.

11. Lenza M, Buchbinder R, Takwoingi Y, Johnston RV, Hanchard NCA, Faloppa F. "Magnetic resonance imaging, magnetic resonance arthrography and ultrasonography for assessing rotator cuff tears in people with shoulder pain for whom surgery is being considered," Cochrane Database of Systematic Reviews. 2013.

12. Handoll $\mathrm{H}$, Hanchard N, Lenza M, Buchbinder R, "Rotator cuff tears and shoulder impingement: a tale of two diagnostic test accuracy reviews," Cochrane Database Syst. Rev., vol. 10, no. October, p. ED000068, 2013.

13. Hanchard NCA, Lenza M, Handoll HHG, Takwoingi Y. "Physical tests for shoulder impingements and local lesions of bursa, tendon or labrum that may accompany impingement," Cochrane Database of Systematic Reviews. 2013.

14. Seida JC, et al., "Systematic review: Nonoperative and operative treatments for rotator cuff tears," Annals of Internal Medicine. 2010.

15. Ainsworth R, Lewis JS. "Exercise therapy for the conservative management of full thickness tears of the rotator cuff: A systematic review," British Journal of Sports Medicine. 2007.

16. Eljabu W, Klinger HM, von Knoch M. "The natural history of rotator cuff tears: a systematic review," Arch. Orthop. Trauma Surg., 2015.

17. Van Der Zwaal P, Thomassen BJW, Nieuwenhuijse MJ, Lindenburg R, Swen JWA, Van Arkel ERA. Clinical outcome in all-arthroscopic versus mini-open rotator cuff repair in small to medium-sized tears: A randomized controlled trial in 100 patients with 1-year follow-up. Arthrosc - J Arthrosc Relat Surg. 2013;29(2):266-73.

18. Morse K, Davis AD, Afra R, Krall Kaye E, Schepsis A, Voloshin I. "Arthroscopic versus mini-open rotator cuff repair: A comprehensive review and meta-analysis," Am. J. Sports Med., 2008. 
19. Ji X, Bi C, Wang F, Wang Q. Arthroscopic versus mini-open rotator cuff repair: An up-to-date metaanalysis of randomized controlled trials. Arthrosc - J Arthrosc Relat Surg. 2015;31(1):118-24.

20. Huang R, Wang S, Wang Y, Qin X, Sun Y. "Systematic Review of All-Arthroscopic Versus Mini-Open Repair of Rotator Cuff Tears: A Meta-Analysis," Scientific Reports. 2016.

21. Adla DN, Rowsell M, Pandey R. "Cost-effectiveness of open versus arthroscopic rotator cuff repair," J. Shoulder Elb. Surg., 2010.

22. Köse K, et al., "Mini-open versus all-arthroscopic rotator cuff repair: Comparison of the operative costs and the clinical outcomes," Adv. Ther., 2008.

23. Vitale MA, Vitale MG, Zivin JG, Braman JP, Bigliani LU, Flatow EL. "Rotator cuff repair: An analysis of utility scores and cost-effectiveness," J. Shoulder Elb. Surg., 2007.

24. Sanders GD, et al., "Recommendations for conduct, methodological practices, and reporting of costeffectiveness analyses: Second panel on cost-effectiveness in health and medicine," JAMA - Journal of the American Medical Association. 2016.

25. Kukkonen J, Kauko T, Vahlberg T, Joukainen A, Äärimaa V. "Investigating minimal clinically important difference for Constant score in patients undergoing rotator cuff surgery," J. Shoulder Elb. Surg., 2013.

26. NCSS L, "PASS 14 Power Analysis and Sample Size Software." Kaysville, Utah, USA, 2015.

27. Harris PA, Taylor R, Thielke R, Payne J, Gonzalez N, Conde JG. "Research electronic data capture (REDCap)-A metadata-driven methodology and workflow process for providing translational research informatics support," J. Biomed. Inform., 2009.

28. Harris PA, et al., "The REDCap consortium: Building an international community of software platform partners," Journal of Biomedical Informatics. 2019.

29. Barreto RPG, Barbosa MLL, Balbinotti MAA, Mothes FC, LHT da Rosa, Silva MF, "Versão brasileira do Constant-Murley Score (CMS-BR): validade convergente e de constructo, consistência interna e unidimensionalidade," Rev. Bras. Ortop., vol. 51, no. 5, pp. 515-520, 2016.

30. Noronha F. "Associação Portuguesa de Economia da Saúde Lara de Noronha e Ferreira," p. 46, 2002.

31. Neto JOB, et al. Validation of the Simple Shoulder Test in a Portuguese-Brazilian Population. Is the Latent Variable Structure and Validation of the Simple Shoulder Test Stable across Cultures? PLoS One. 2013;8(5):1-8.

32. Ludbrook J. "PRACTICAL STATISTICS FOR MEDICAL RESEARCH," Australian and New Zealand Journal of Surgery. 1991.

33. Faraway JJ. Extending the linear model with R: generalized linear, mixed effects and nonparametric regression models. 2006.

34. IBM Corp., "IBM SPSS Statistics for Windows, Version 24.0," 2016. 2016.

35. Hui YJ, Teo AQA, Sharma S, Tan BHM, Prem Kumar V. Immediate costs of mini-open versus arthroscopic rotator cuff repair in an Asian population. J Orthop Surg. 2017;25(1):1-6. 
36. Churchill RS, Ghorai JK. "Total cost and operating room time comparison of rotator cuff repair techniques at low, intermediate, and high volume centers: Mini-open versus all-arthroscopic," J. Shoulder Elb. Surg., 2010.

37. Carr AJ, et al., "Clinical effectiveness and cost-effectiveness of open and arthroscopic rotator cuff repair [the UK rotator cuff surgery (UKUFF) randomised trial]," Health Technol. Assess. (Rockv)., 2015.

\section{Tables}

Table1. Timetable of assessment

\begin{tabular}{|c|c|c|c|c|c|c|c|c|}
\hline \multirow[b]{3}{*}{ TIMEPOINT** } & \multicolumn{8}{|c|}{ STUDY PERIOD } \\
\hline & \multirow{2}{*}{$\begin{array}{l}\text { Enrolment } \\
0\end{array}$} & \multirow{2}{*}{$\begin{array}{l}\text { Allocation } \\
0\end{array}$} & \multicolumn{5}{|c|}{ Post-allocation } & \multirow{2}{*}{$\begin{array}{l}\text { Close-out } \\
48 w\end{array}$} \\
\hline & & & $1 w$ & $2 w$ & $6 w$ & $24 w$ & $48 w$. & \\
\hline \multicolumn{9}{|l|}{ ENROLMENT: } \\
\hline Eligibility screen & $x$ & & & & & & & \\
\hline Informed consent & $x$ & & & & & & & \\
\hline CM;EQ-5D ; SST; VAS & $x$ & & & & & & & \\
\hline Allocation & & $x$ & & & & & & \\
\hline \multicolumn{9}{|l|}{ INTERVENTIONS: } \\
\hline Open Repair & & & $x$ & & & & & \\
\hline [Arthroscopic Repari] & & & $x$ & & $x$ & & & \\
\hline \multicolumn{9}{|l|}{ ASSESSMENTS: } \\
\hline CM;EQ-5D ; SST; VAS & $\mathrm{x}$ & & $x$ & $x$ & $x$ & $x$ & $x$ & $x$ \\
\hline CM;EQ-5D ; SST; VAS & & & $x$ & $\mathrm{x}$ & $\mathrm{x}$ & $x$ & $\mathrm{x}$ & $\mathrm{x}$ \\
\hline Economics & & & $\mathrm{x}$ & $\mathrm{x}$ & $\mathrm{x}$ & $x$ & $\mathrm{x}$ & $\mathrm{x}$ \\
\hline MRI & $x$ & & & & & & & $x$ \\
\hline Complications & & & $x$ & $x$ & $x$ & $x$ & $x$ & $x$ \\
\hline
\end{tabular}

\section{Figures}




\section{PATIENT FLOW CHART}

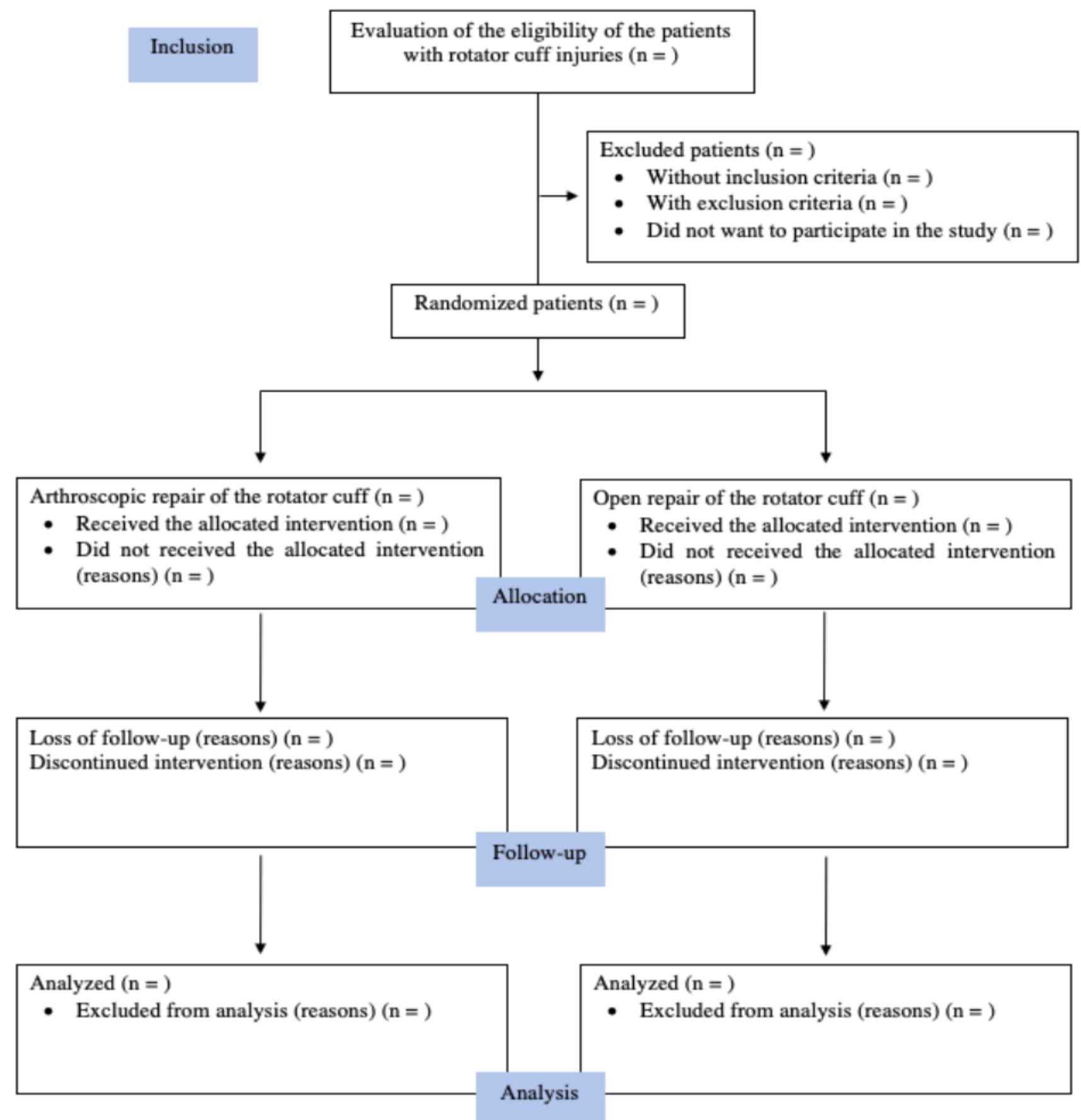

\section{Figure 1}

Flow of participants. The diagram shows the planned flow of participants through each stage of the study.

\section{Supplementary Files}


This is a list of supplementary files associated with this preprint. Click to download.

- SPIRITChecklistECRAlvorada.doc

- CONSORTCHECKLIST.doc 